\title{
MULTIBAND VLA OBSERVATIONS OF THE FAINT RADIO CORE OF 3CR 68.1
}

\author{
Michael S. Brotherton, ${ }^{1}$ Chun Ly, ${ }^{2}$ Beverley J. Wills, ${ }^{3}$ Sally A. Laurent-Muehleisen, ${ }^{4}$ \\ Wil VAN BREugel, ${ }^{5}$ AND R. R. J. ANTONUCCI ${ }^{6}$ \\ Received 2002 May 31; accepted 2002 June 18
}

\begin{abstract}
The large, powerful Fanaroff-Riley class II radio source 3CR 68.1 is an optically red quasar with strong evidence of dust reddening, intrinsic ultraviolet line absorption, and X-ray absorption. In the context of its large, extended radio structure and the evidence for intrinsic material along the line of sight, it is a good candidate for a quasar seen through the edge of an obscuring torus, as proposed by unified schemes. The compact radio core coincident with the optical position of the quasar is extremely weak compared with the very bright and luminous radio lobes, suggesting that the radio core might also suffer absorption. We observed 3CR 68.1 at six frequencies and show that the radio core has a flat spectrum with no evidence of free-free absorption or other strong absorption mechanisms. This result helps establish an empirical intrinsic lower range for core-to-lobe radio flux in the most powerful radio sources.
\end{abstract}

Key words: quasars: general — quasars: individual (3CR 68.1) — radio continuum

\section{INTRODUCTION}

The extreme properties of 3CR 68.1 indicate that it is seen in an edge-on geometry (Brotherton et al. 1998), along a line of sight through dusty, ionized gas, perhaps part of an obscuring torus (see, e.g., Antonucci 1993). Such a structure seen in such a geometry helps to explain the dust-reddened optical continuum $\left(A_{V}=1.7\right.$ mag; Simpson \& Rawlings 2000), scattered light leading to a rising continuum polarization up to $13 \%$ at $2000 \AA$ rest-frame (Brotherton et al. 1998), intrinsic ultraviolet absorption lines including metastable He I* (Brotherton et al. 1998), extremely weak and probably absorbed X-ray emission (Bregman et al. 1985), and the large radio lobe separation $\left(53^{\prime \prime}\right.$ at $z=1.228$ ) and small radio core dominance (Bridle et al. 1994) that mark an edge-on radio structure (see, e.g., Orr \& Browne 1982).

The radio core of 3CR 68.1 is an order of magnitude fainter than that of any other $3 \mathrm{CR}$ quasar $(1.1 \mathrm{mJy}$ at $5 \mathrm{GHz}$; Bridle et al. 1994), and it is weak even among Fanaroff-Riley type II (FR II; Fanaroff \& Riley 1974) radio galaxies. At the same time, the radio lobes make 3CR 68.1 one of the three most powerful 3CR quasars at $178 \mathrm{MHz}$ in the compilation by Laing, Riley, \& Longair (1983). We hypothesized that the radio core might be so faint because it suffers free-free absorption from the obscuring material along the line of sight, measurements

\footnotetext{
${ }^{1}$ Kitt Peak National Observatory, National Optical Astronomy Observatory, 950 North Cherry Avenue, P.O. Box 26732, Tucson, AZ 85726; mbrother@noao.edu. Current address: Department of Physics and Astronomy, University of Wyoming, P.O. Box 3905, Laramie, WY 82071 .

${ }^{2}$ Steward Observatory, University of Arizona, 933 North Cherry Avenue, Tucson, AZ 85721-0065.

${ }^{3}$ McDonald Observatory and Department of Astronomy, RLM 15.308, University of Texas at Austin, Austin, TX 78712.

${ }^{4}$ Department of Physics, University of California, Davis, 1 Shields Avenue, Davis, CA 95616.

${ }^{5}$ Institute of Geophysics and Planetary Physics, Lawrence Livermore National Laboratory, L-413, P.O. Box 808, Livermore, CA 94550.

${ }^{6}$ Department of Physics, University of California, Santa Barbara, Santa Barbara, CA 93106-9530.
}

of which would provide an additional probe of its physical conditions.

We obtained multiband observations of 3CR 68.1 using the $\mathrm{NRAO}^{7}$ Very Large Array (VLA) in order to test this hypothesis. Following sections of this paper discuss the observations and data reduction $(\S 2)$, measurements of the core $(\S 3)$, and our null result and its implications $(\S 4)$. The final section ( $(5)$ summarizes our conclusions.

\section{OBSERVATIONS AND DATA REDUCTION}

We observed 3CR 68.1 with the VLA in its A configuration on 1998 May 24 (program AW482), using six frequencies: $325 \mathrm{MHz}(90 \mathrm{~cm}, \mathrm{P}$ band), $1.4 \mathrm{GHz}(20 \mathrm{~cm}, \mathrm{~L}$ band), $4.8 \mathrm{GHz}(6 \mathrm{~cm}, \mathrm{C}$ band $), 8.4 \mathrm{GHz}(3.6 \mathrm{~cm}, \mathrm{X}$ band $), 15 \mathrm{GHz}$ $(2.0 \mathrm{~cm}, \mathrm{U}$ band), and $22 \mathrm{GHz}(1.3 \mathrm{~cm}, \mathrm{~K}$ band). Observations at each frequency were interleaved to provide appropriate sampling of the $u-v$ plane. We observed $3 \mathrm{C} 48$ as the primary flux calibrator and several phase calibrators between observations of 3CR 68.1. Table 1 gives the observing log, showing the total observing time per source at each frequency.

Calibrations were done in AIPS and in general followed standard recipes in the AIPS Cookbook. ${ }^{8}$ Flux calibration at $15 \mathrm{GHz}$ for $3 \mathrm{C} 48$ required small modification in the $u-v$ range compared with suggested values. For 22 and $8.4 \mathrm{GHz}$, we calibrated with clean-component models using the task CALIB. We have not performed a polarization calibration (the radio core is unpolarized; Bridle et al. 1994). Following calibration we used MAPIT to self-calibrate and produce CLEAN maps of our source. Figures $1-3$ show these images as contour maps. Figure 1 shows only the central core region along with the beam, as the lobes are weak or resolved out at these high frequencies.

\footnotetext{
${ }^{7}$ The National Radio Astronomy Observatory is a facility of the National Science Foundation operated under cooperative agreement by Associated Universities, Inc.

${ }^{8}$ See http://www.aoc.nrao.edu/aips/cook.html.
} 
TABLE 1

VLA A-Configuration Observation Log (1998 May 24)

\begin{tabular}{|c|c|c|c|c|c|c|}
\hline Object & $\begin{array}{l}22 \mathrm{GHz} \\
\text { (s) }\end{array}$ & $\begin{array}{l}15 \mathrm{GHz} \\
\text { (s) }\end{array}$ & $\begin{array}{l}8.4 \mathrm{GHz} \\
\text { (s) }\end{array}$ & $\begin{array}{l}4.8 \mathrm{GHz} \\
\text { (s) }\end{array}$ & $\begin{array}{l}1.4 \mathrm{GHz} \\
\text { (s) }\end{array}$ & $\begin{array}{c}0.3 \mathrm{GHz} \\
\text { (s) }\end{array}$ \\
\hline $0137+331(3 \mathrm{C} 48) \ldots \ldots \ldots \ldots . .$. & 70 & 50 & 60 & 60 & 80 & 150 \\
\hline $0237+288 \ldots \ldots \ldots \ldots \ldots$ & 550 & 550 & 660 & 620 & 460 & 730 \\
\hline 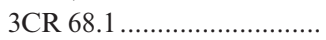 & 1250 & 1500 & 1060 & 1110 & 1240 & 1190 \\
\hline $0319+415 \ldots \ldots \ldots \ldots \ldots \ldots \ldots$ & 60 & 60 & 70 & 70 & 90 & $\ldots$ \\
\hline $0521+166 \ldots \ldots \ldots \ldots \ldots \ldots \ldots \ldots$ & 60 & 60 & 70 & 60 & 80 & 80 \\
\hline
\end{tabular}

\section{MEASUREMENTS AND THE RADIO CORE SPECTRUM}

The optical position of 3 CR 68.1 is R.A. $=02^{\mathrm{h}} 32^{\mathrm{m}} 28.9$, decl. $=+34^{\circ} 23^{\prime} 47^{\prime \prime}(\mathrm{J} 2000)$. We detect a radio point source at that position, the radio core, in all our total intensity maps except at $325 \mathrm{MHz}$. We used the AIPS task JMFIT to measure the position and flux density of the radio core. Table 2 provides these measurements. The rms values in the table are from JMFIT. The uncertainties on the flux were computed by adding in quadrature the rms noise in the map, plus the reported error in the fit (from JMFIT) and an assumed 3.33\% error. The uninteresting $325 \mathrm{MHz}$ limit comes from running IMSTAT to determine the peak flux in a small box around the core position; a more conservative limit is 5 times the rms noise, or $65 \mathrm{mJy}$. The actual 325 $\mathrm{MHz}$ flux of the core is likely about $1 \mathrm{mJy}$ or less. Figure 4 plots these peak flux densities against frequency to show the radio spectrum of the core. The most accurate position for the radio core measured to date comes from our highfrequency maps and is $02^{\mathrm{h}} 32^{\mathrm{m}} 28^{\mathrm{s}} .88,+34^{\circ} 23^{\prime} 46^{\prime \prime} \cdot 7(\mathrm{~J} 2000)$.

\section{RESULTS AND DISCUSSION}

We have detected the radio core of 3CR 68.1, coincident with the optical position, at five of six frequencies. Our total intensity map at $4.8 \mathrm{GHz}$ (Fig. 2, right) is consistent with the deeper 4.8 GHz map presented by Bridle et al. (1994), who label the radio core component " $E$ " and report it to be 1.1 $\mathrm{mJy}$. This is essentially the same as the value we measure, $1.05 \pm 0.16 \mathrm{mJy}$ (Table 2). There is therefore no evidence of significant variability during the 12 years between observations.

Figure 4 shows that the radio core spectrum has a normal flat spectrum from 1.4 to $22 \mathrm{GHz}$, observed frame, or 3-50 $\mathrm{GHz}$ rest-frame $(z=1.228)$, with a flux density of $\sim 1 \mathrm{mJy}$. The nondetection at the lowest frequency used, $325 \mathrm{MHz}$, leaves us with an uninteresting upper limit; there could be absorption at this frequency, but we have no evidence for this. So, what is the significance of the null result for absorption in the radio core?

It was this weakness of the radio core at $5 \mathrm{GHz}$ relative to the thousand times brighter radio lobes $(0.83 \mathrm{Jy})$ that in part suggested that the core might be absorbed at this frequency. ${ }^{9}$ An observed-frame frequency of $5 \mathrm{GHz}$ corresponds to $11 \mathrm{GHz}$ in the rest frame of 3CR 68.1. Using a radio spectral index of -1 to characterize the lobe spectrum and a flat radio core spectrum then implies a $K$-corrected core dominance parameter at $5 \mathrm{GHz}$ rest-frame of $\log R=-3.3$, where $R=S_{\text {core }} / S_{\text {lobe }}$ (Orr \& Browne 1982).

\footnotetext{
${ }^{9}$ The Bridle et al. (1994) core detection at $5 \mathrm{GHz}$ is the only such measurement in the literature at any radio frequency for 3CR 68.1.
}
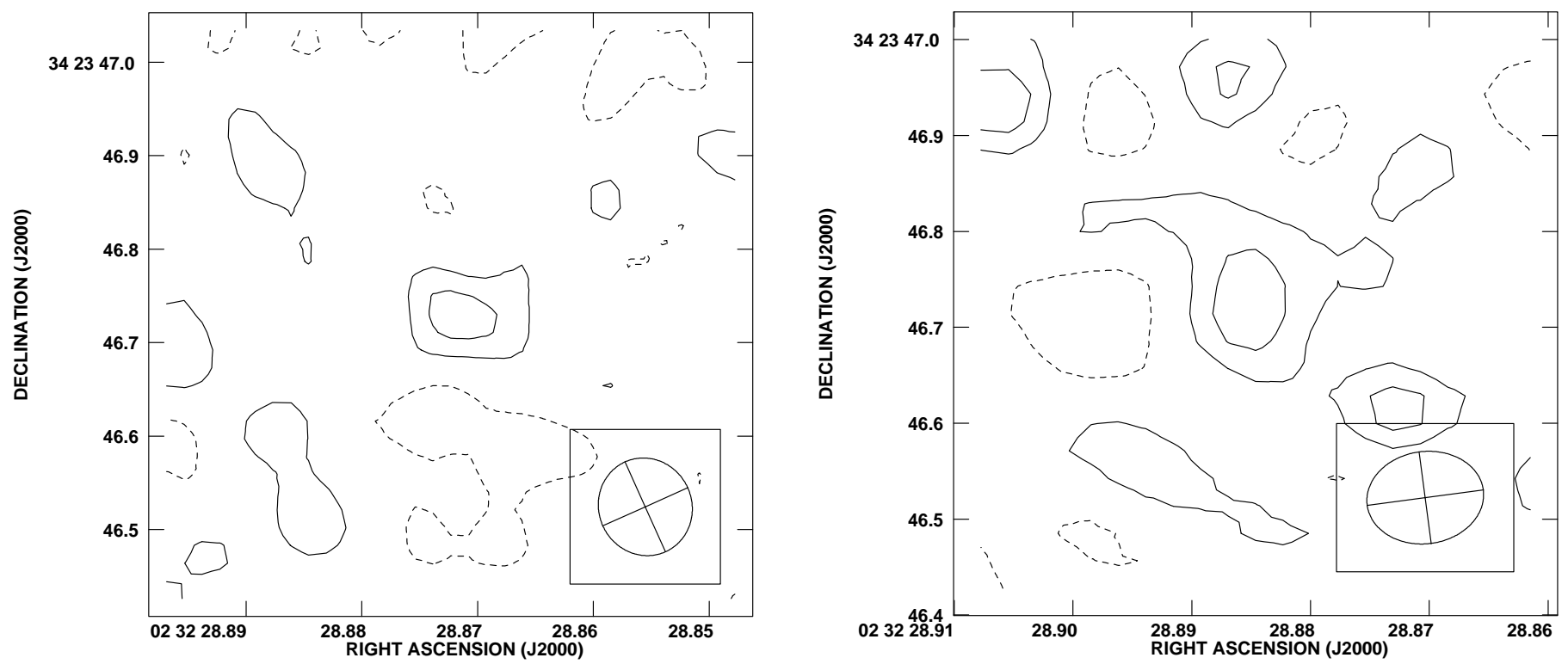

FIG. 1.-Total intensity contour maps of the $3 \mathrm{CR} 68.1$ core region at $22 \mathrm{GHz}$ (K band, left) and $15 \mathrm{GHz}$ (U band, right). Beams are plotted to show the resolution of the array. Contour levels are drawn at 2.5, 5, 10,20, etc., times the rms flux as given in Table 2. The maps are centered on the core. 

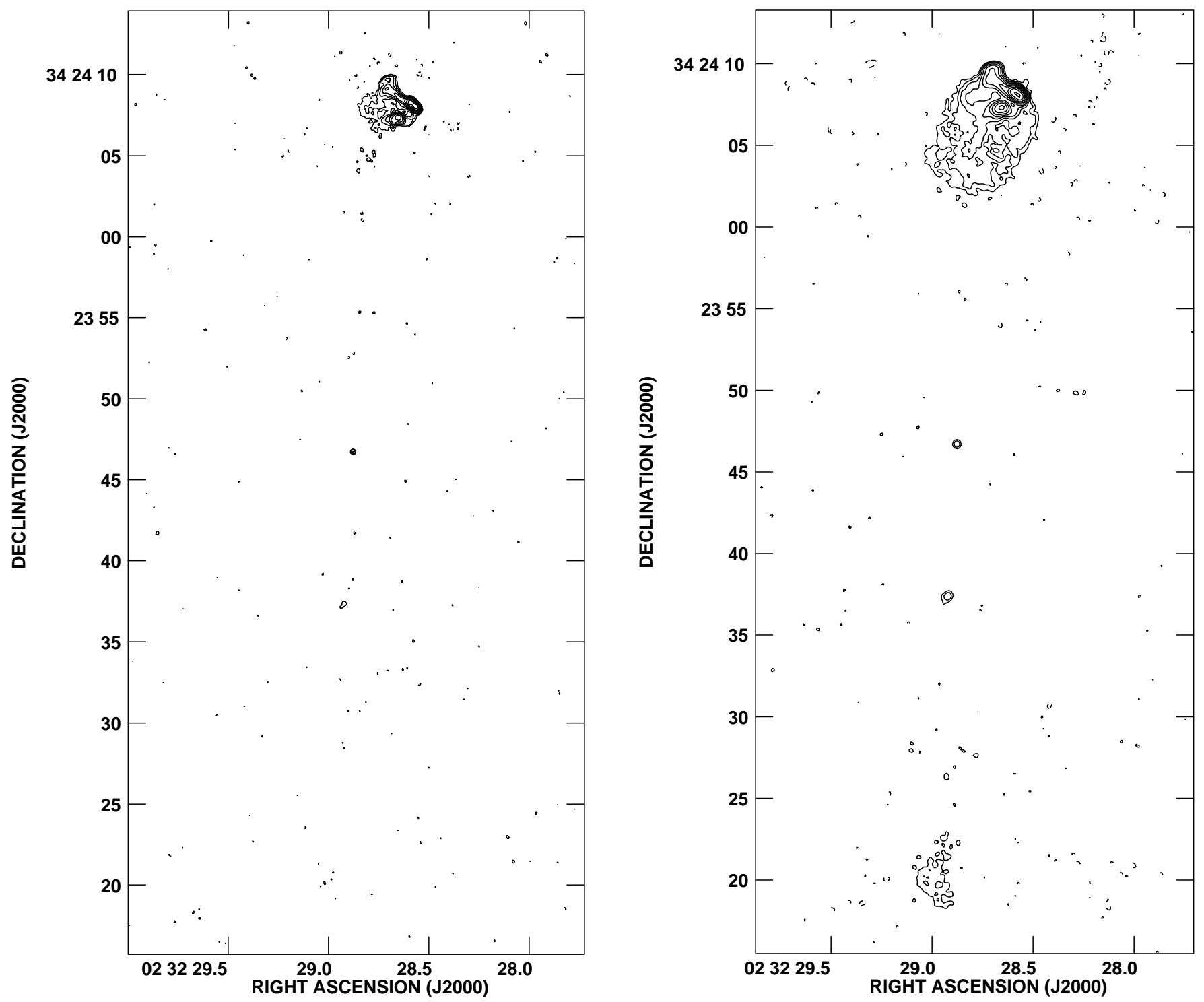

FIG. 2.- Total intensity contour maps of 3CR 68.1 at $8.4 \mathrm{GHz}$ (X band, left) and $4.8 \mathrm{GHz}(\mathrm{C}$ band, right). Contour levels are drawn at 2.5, 5, 10, 20, etc., times the rms flux as given in Table 2 .

This value is extremely small, an order of magnitude smaller than that of other very lobe-dominated quasars (see, e.g., Wills \& Browne 1986). For reference, the nearby edge-on radio galaxy Cygnus A shows a ratio of core to lobe flux at $5 \mathrm{GHz}$ of $0.5 \%$, or $\log R=-2.3$.

The line-of-sight optical reddening toward 3CR 68.1 is on the order of $A_{V}=1.7 \mathrm{mag}$ (Simpson \& Rawlings

TABLE 2

Radio CORE FluXes For 3CR 68.1

\begin{tabular}{|c|c|c|c|}
\hline Band & $\begin{array}{c}\text { Frequency } \\
\quad(\mathrm{GHz})\end{array}$ & $\begin{array}{l}\text { Flux } \\
(\mathrm{mJy})\end{array}$ & $\begin{array}{c}\mathrm{rms} \\
(\mathrm{mJy})\end{array}$ \\
\hline .............. & 0.3 & $\leq 8$ & 13 \\
\hline L ...................... & 1.4 & $0.65 \pm 0.42$ & 0.29 \\
\hline С ..................... & 4.8 & $1.05 \pm 0.15$ & 0.11 \\
\hline $\mathrm{X}$ & 8.4 & $1.04 \pm 0.07$ & 0.09 \\
\hline $\mathrm{U}$ & 15 & $0.89 \pm 0.28$ & 0.11 \\
\hline $\mathrm{K}$ & 22 & $0.69 \pm 0.24$ & 0.09 \\
\hline
\end{tabular}

2000) or perhaps slightly smaller, depending on the host galaxy's contribution (Brotherton et al. 1998). Using an estimate of $E(B-V)=0.5$ then corresponds to a gas column density of $N_{\mathrm{H}}=3 \times 10^{21} \mathrm{~cm}^{-2}$, given the standard gas-to-dust ratio for the Galaxy. A Small Magellanic Cloud (SMC) extinction law is more consistent with the apparent reddening toward 3CR 68.1, and the SMC has a larger gas-to-dust ratio, implying a larger value of $N_{\mathrm{H}}=3 \times 10^{22} \mathrm{~cm}^{-2}$. Gas-to-dust ratios toward quasars may surpass Galactic values by as much as 2 orders of magnitude (Maiolino et al. 2001), so an associated gas column density of $N_{\mathrm{H}} \sim 10^{22} \mathrm{~cm}^{-2}$ is probably a rough order-of-magnitude lower limit.

If the obscuring, outflowing gas along the line of sight toward 3CR 68.1 is local to the environment of the nuclear activity, as is generally believed for warm absorbers, the material would be photoionized with temperatures of $T_{e} \sim 10^{4} \mathrm{~K}$

In the radio regime, the free-free opacity is a function of frequency, temperature, and the emission measure. From 

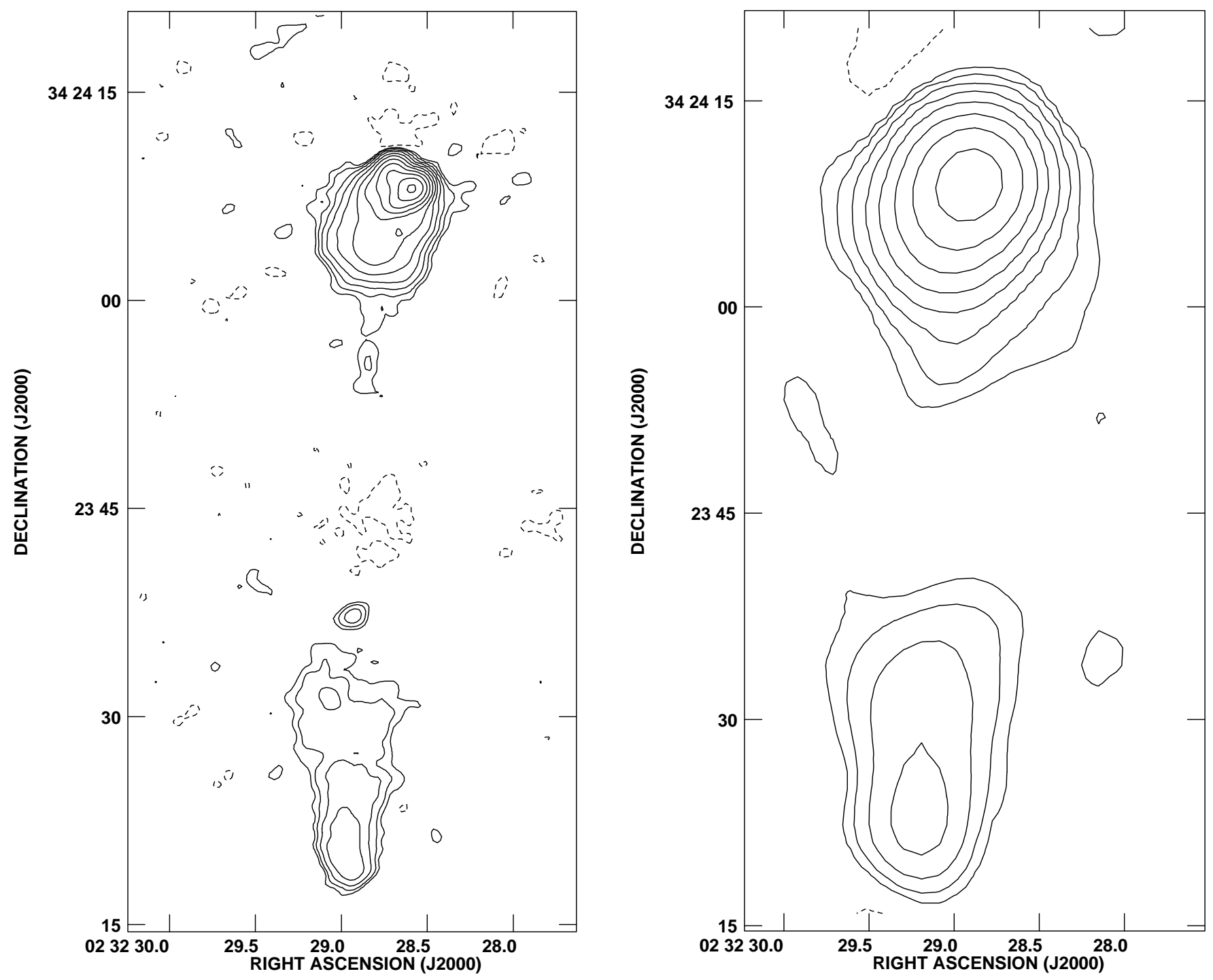

FIG. 3.- Total intensity contour maps of 3CR 68.1 at $1.4 \mathrm{GHz}$ (L band, left) and $325 \mathrm{MHz}$ (P band, right). Contour levels are drawn at 2.5, 5, 10, 20, etc., times the rms flux as given in Table 2 .

Osterbrock (1989),

$$
\tau_{\mathrm{ff}}=8.24 \times 10^{-2} T^{-1.35} \nu^{-2.1} E,
$$

where $T$ is the temperature in kelvins, $\nu$ is in gigahertz, and $E$ is the emission measure in $\mathrm{cm}^{-6}$ pc. Free-free absorption is insignificant at high frequencies, but it becomes strong at frequencies lower than the turnover frequency at which $\tau_{\text {ff }}=1$. This sharp turnover signifies free-free absorption and indicates a column of ionized gas along the line of sight. For the redshift of 3CR 68.1 and a photoionization temperature of $T_{e} \sim 10^{4} \mathrm{~K}$, equation (1) can be rewritten to estimate the turnover frequency:

$$
\nu_{0} \approx 15\left(n_{e, 5} N_{\mathrm{H}, 22}\right)^{0.5} \mathrm{GHz},
$$

where $n_{e .5}$ is the electron density in units of $10^{5} \mathrm{~cm}^{-3}$ and $N_{\mathrm{H}, 22}$ is the ionized hydrogen column in units of $10^{22}$ atoms $\mathrm{cm}^{-2}$. Given that the total hydrogen column density is on the order of $10^{22} \mathrm{~cm}^{-2}$ or larger, we might well have expected to see free-free absorption in the frequency range we have investigated here. Lines of sight through an obscuring torus are similarly predicted to show free-free absorption in the range of a few to $10 \mathrm{GHz}$ rest-frame (e.g., Krolik \& Lepp 1989; Neufeld, Maloney, \& Conger 1994). Free-free absorption has been seen in some edge-on systems with warm absorbers, for instance, NGC 4151 (Pedlar et al. 1998), but not other edge-on systems (e.g., Barvainis \& Lonsdale 1998).

There are several possible explanations why we do not see free-free absorption in 3CR 68.1 despite the above argument. First, it may be that the radio core comes from a larger region than the optical continuum and is not significantly covered by the outflow. Also possible is that the outflow is of very low ionization ( $\mathrm{Mg}$ I $\lambda 2853$ is present) and that the column density of ionized gas is too low to cause free-free absorption at these frequencies; this might suggest that the outflow is not close to the continuum source.

So if the radio core is not absorbed, could the extremely low core dominance arise from overluminous radio lobes? After all, the radio lobes reflect a previous epoch of activity that may not be representative of the current activity level in 


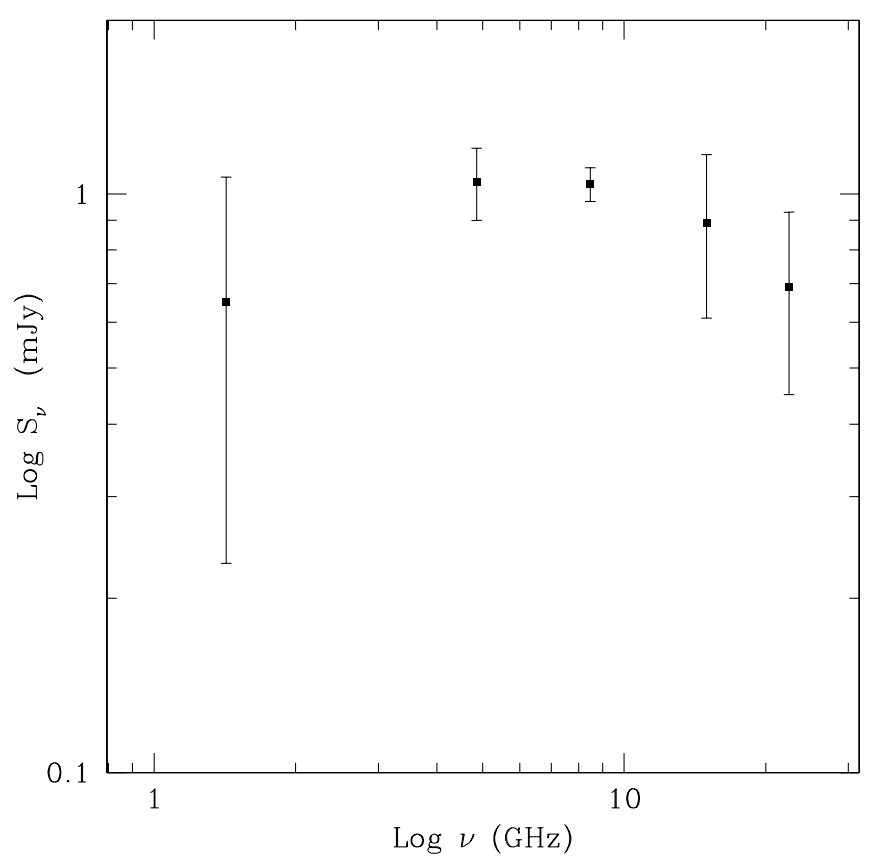

FIG. 4.-The 3CR 68.1 core flux density $S_{\nu}$ vs. frequency $\nu$, observed frame, with uncertainties described in the text and given in Table 2.

the core. Furthermore, radio lobe brightness does not depend solely upon properties of the central engine, but also environmental factors. This question can be addressed by looking at the value of the alternative core-dominance parameter $R_{V}$, which normalizes the radio core using the optical continuum (Wills \& Brotherton 1995). Using a dereddened absolute magnitude $M_{B}=-28.2$ (for $H_{0}=50 \mathrm{~km}$ $\mathrm{s}^{-1} \mathrm{Mpc}^{-1}$ and $q_{0}=0$; Brotherton et al. 1998) and a radio core luminosity of $2 \times 10^{24} \mathrm{~W} \mathrm{~Hz}{ }^{-1}$, then $\log R_{V}=$ $\log L_{\text {core }}+M_{B} / 2.5-13.69$, or $\log R_{V}=-0.7$ in the case of 3CR 68.1. Comparison with the distribution of $\log R_{V}$ for representative bright quasars (Brotherton 1996) shows that once again the radio core of 3CR 68.1 is underluminous for its optical luminosity by at least an order of magnitude. We conclude that the radio core truly is intrinsically very faint despite the high optical and radio lobe luminosities of $3 \mathrm{CR}$ 68.1. Anomalously strong Doppler debeaming could be responsible for the radio core faintness given a faster-thanusual jet, for instance, but the faintness at gigahertz frequencies is not the result of absorption from material associated with the absorbers present in other wave bands. Thus, 3CR 68.1 apparently represents some kind of natural extreme for radio core-to-lobe ratios in quasars.

\section{CONCLUSIONS}

While 3CR 68.1 clearly has dust and gas along the line of sight that causes absorption at optical and X-ray wavelengths, there does not appear to be any significant absorption toward the radio core at gigahertz frequencies. The radio core's spectrum is consistent with a flat $(\alpha=0)$ spectrum of approximately $1 \mathrm{mJy}$, less than $1 / 1000$ as bright as the radio lobes at $5 \mathrm{GHz}$, rest frame, with no evidence for free-free or other absorption at radio frequencies. The case of 3CR 68.1 apparently represents the intrinsic low extreme of the radio core dominance parameter used to characterize orientation in unified schemes.

We thank Tony Beasley for help constructing an effective observing program, and an anonymous referee for suggestions that improved this paper. The National Optical Astronomy Observatory is operated by the Association of Universities for Research in Astronomy, Inc., under cooperative agreement with the National Science Foundation. This research has made use of the NASA/IPAC Extragalactic Database, which is operated by the Jet Propulsion Laboratory, California Institute of Technology, under contract with the National Aeronautics and Space Administration. The work by W. v. B. at the Institute of Geophysics and Planetary Physics at Lawrence Livermore National Laboratory was performed under the auspices of the US Department of Energy by the University of California, Lawrence Livermore National Laboratory, under contract W-7405ENG-48.

\section{REFERENCES}

Antonucci, R. 1993, ARA\&A, 31, 473

Barvainis, R., \& Lonsdale, C. 1998, AJ, 115, 885

Bregman, J. N., Glassgold, A. E., Huggins, P. J., \& Kinney, A. L. 1985, ApJ, 291, 505

Bridle, A. H., Hough, D. H., Lonsdale, C. J., Burns, J. O., \& Laing, R. A. 1994, AJ, 108, 766

Brotherton, M. S. 1996, ApJS, 102, 1

Brotherton, M. S., Wills, B. J., Dey, A., van Breugel, W., \& Antonucci, R. 1998, ApJ, 501, 110

Fanaroff, B. L., \& Riley, J. M. 1974, MNRAS, 167, 31P

Krolik, J. H., \& Lepp, S. 1989, ApJ, 347, 179

Laing, R. A., Riley, J. M., \& Longair, M. S. 1983, MNRAS, 204, 151
Maiolino, R., Marconi, A., Salvati, M., Risaliti, G., Severgnini, P., Oliva, E. La Franca, F. \& Vanzi, L. 2001, A\&A, 365, 28

Neufeld, D. A., Maloney, P. R., \& Conger, S. 1994, ApJ, 436, L127

Orr, M. J. L., \& Browne, I. W. A. 1982, MNRAS, 200, 1067

Osterbrock, D. E. 1989, Astrophysics of Gaseous Nebulae and Active Galactic Nuclei (Mill Valley, CA: Univ. Sci.)

Pedlar, A., Fernandez, B., Hamilton, N. G., Redman, M. P., \& Dewdney, P. E. 1998, MNRAS, 300, 1071

Simpson, C., \& Rawlings, S. 2000, MNRAS, 317, 1023

Wills, B. J., \& Brotherton, M. S. 1995, ApJ, 448, L81

Wills, B. J., \& Browne, I. W. A. 1986, ApJ, 302, 56 